\title{
Epigenetic silencing of BCL6B inactivates p53 signaling and causes human hepatocellular carcinoma cell resist to 5-FU
}

\author{
Xin Li ${ }^{1,4}$, Jie Yu ${ }^{1}$, Malcolm V. Brock ${ }^{2}$, Qian Tao ${ }^{3}$, James G. Herman ${ }^{2}$, Ping Liang ${ }^{1}$, \\ Mingzhou Guo ${ }^{4}$ \\ ${ }^{1}$ Department of Interventional Ultrasound, Chinese PLA General Hospital, Beijing, China \\ ${ }^{2}$ Oncology Center, Johns Hopkins University, Baltimore, Maryland, USA \\ ${ }^{3}$ Department of Clinical Oncology, The Chinese University of Hong Kong, Hong Kong, China \\ ${ }^{4}$ Department of Gastroenterology \& Hepatology, Chinese PLA General Hospital, Beijing, China
}

Correspondence to:

Mingzhou Guo, e-mail: mzguo@hotmail.com

Ping Liang, e-mail: liangping301@hotmail.com

James G. Herman, e-mail: hermaji@jhmi.edu

Keywords: BCL6B, DNA methylation, hepatocellular carcinoma, p53 signaling, 5-fluorouracil

Received: October 29, $2014 \quad$ Accepted: February 19, $2015 \quad$ Published: March 21, 2015

\section{ABSTRACT}

BCL6B is a potential tumor suppressor in human gastric cancer, but the regulation and mechanism of BCL6B in human hepatocellular carcinogenesis remain unclear. This study is to explore the epigenetic change and mechanism of BCL6B in human hepatocellular carcinoma (HCC). Nineteen hepatic cancer cell lines, 50 cases of adjacent tissue and 149 cases of HCC samples were employed. BCL6B is methylated in $100 \%(19 / 19)$ of human HCC cell lines, $40.0 \%(20 / 50)$ of adjacent tissue samples and $86.6 \%(129 / 149)$ of primary cancer samples. Methylation of BCL6B is associated with HBV positive $(p<0.05)$. But no association was found with age, sex, tumor size, differentiation, TNM stage, recurrence and survival. Loss of BCL6B expression was found in 19 of completely methylated HCC cell lines. BCL6B was re-expressed after 5-aza-2'-deoxycytidine treatment. Restoration of BCL6B expression suppressed cell proliferation, induced apoptosis and G1/S arrest in HCC cells. The expression of EGR1, a key component of p53 signaling, was increased after re-expression BCL6B in HCC cells. Re-expression of BCL6B activated p53 signaling and sensitized HCC cells to 5-fluorouracil. BCL6B is frequently methylated in human HCC and the expression of BCL6B is regulated by promoter region hypermethylation. BCL6B activates p53 signaling by increasing EGR1 expression in HCC.

\section{INTRODUCTION}

Hepatocellular carcinoma (HCC) is the fifth most malignant cancer and the third leading cause of cancer-related death worldwide $[1,2]$. Even through improved interventional and surgical treatment, The 5 -year survival rate is less than $15 \%$. The major reason is cancer recurrence and metastasis [3-5]. Emerging evidence indicates that epigenetic alterations, particularly inactivation of tumor suppressor genes or tumor-related genes via promoter region hypermethylation, play an important role in the development and progression of $\mathrm{HCC}$ [6-9]. B cell CLL/lymphoma 6 member B (BCL6B) is a homologue of B cell CLL/lymphoma 6 (BCL6). It is located on chromosome 17p13.1, a region of $800 \mathrm{bp}$ next to $\mathrm{p} 53$ gene. BCL6B was regarded as a potential tumor suppressor and methylation of BCL6B may serve as a poor prognosis marker in gastric cancer [10-12]. In this study, we analyzed the epigenetic changes and the mechanism of BCL6B in hepatocellular carcinogenesis.

\section{RESULTS}

BCL6B expression was regulated by promoter region hypermethylation in $\mathrm{HCC}$ cell lines

To explore the expression of BCL6B in HCC cells, semi-quantitative RT-PCR was employed. Loss of BCL6B 
expression was found in HepG2, SNU449, PLC/PRF/5, SK-Hep1, SMMC-7721, HBXF344, LAM3, SNU182, SNU387, SNU475, Hep3B, Bel-7402, QSG7701, QGY7703, BEL-7404, BEL-7405, MHCC97H, Huh7 and Huh1 cells (Figure 1A). The promoter region methylation was detected by MSP. MSP primers were designed around transcription start site in the $\mathrm{CpG}$ island of BCL6B gene promoter region. Complete methylation was found in HepG2, SNU449, PLC/PRF/5, SK-Hep1, SMMC-7721, HBXF344, LAM3, SNU182, SNU387, SNU475, Hep3B, Bel-7402, QSG7701, QGY-7703, BEL-7404, BEL7405, MHCC97H, Huh7 and Huh1 cells (Figure 1B). To further validate MSP results and the density of promoter region methylation, bisulfite sequencing was performed in HepG2, SNU449, Bel-7402 and HBXF344 cells. As shown in Figure 1C, MSP results are correlated with bisulfite sequencing results very well. HepG2, SNU449, Bel-7402 and HBXF344 cells are densely methylated. These results suggest that BCL6B expression is correlated with methylation status in HCC cells. To further validate the expression of BCL6B is regulated by promoter region hypermethylation, 5-Aza, a DNA methylation transferase inhibitor, was applied in these HCC cells. Re-expression of BCL6B was found in HepG2, SNU449, PLC/PRF/5, SK-Hep1, SMMC-7721, HBXF344, LAM3, SNU182, SNU387, SNU475, Hep3B, Bel-7402, QSG7701, QGY-7703, BEL-7404, BEL7405, MHCC97H, Huh7 and Huh1 cells (Figure 1A). These results further suggest that BCL6B expression was regulated by promoter region methylation in HCC.

\section{BCL6B is frequently methylated in human primary $\mathrm{HCC}$ and reduced expression was associated with promoter region hypermethylation}

To investigate the methylation status of BCL6B, 149 cases of primary HCC, 50 cases of cancer adjacent tissue and 8 cases of normal liver tissue samples were examined by MSP. As shown in Figure 1B \& 1C, 86.6\% (129/149) of HCC and 40.0\% (20/50) of adjacent tissue samples were methylated, but no methylation was found in normal liver tissue samples. Methylation of BCL6B is associated with HBV positive HCC $(p<0.05)$. No association was found between BCL6B methylation and hepatitis in adjacent tissue samples $(p>0.05)$. Existing hepatitis was evaluated by elevated the level of aspartate aminotransferase (AST) and alanine aminotransferase (ALT). No association was found between BCL6B methylation and age, gender, tumor size, cell differentiation and TNM stage (Table 1). The expression of BCL6B was evaluated by IHC in 30 cases of available $\mathrm{HCC}$ and matched adjacent tissue samples. Reduced expression was found in 21 cases of cancer samples and 9 cases of adjacent tissue samples (Figure 2A). Reduced expression of BCL6B is significantly in cancer tissue compared with adjacent tissue samples (Figure 2B, $p<0.05$ ). In 21 cases of BCL6B reduced cancer samples, 18 cases were methylated (Figure 2C). Reduced expression was associated with promoter region hypermethylation significantly (Figure $2 \mathrm{D}, p<0.01$ ). It suggests that BCL6B is possibly regulated by promoter region methylation in human primary $\mathrm{HCC}$.

\section{Restoration of BCL6B expression suppresses cell proliferation, induces apoptosis and $\mathrm{G1} / \mathrm{S}$ arrest in $\mathrm{HCC}$ cells}

The effect of BCL6B on cell proliferation was analyzed by colony formation. The colony number was $502.67 \pm 50.01$ vs. $118.67 \pm 32.08$ in HepG2 cells $(p<0.01)$ and $506.67 \pm 90.89$ vs. $198.67 \pm 33.31$ in SNU449 cells $(p<0.01)$ before and after restoration of BCL6B expression (Figure 3A). The results suggest that HCC cell colony formation was suppressed by BCL6B. The cell viability was detected by MTT. The OD value is $1.174 \pm 0.058$ vs. $0.687 \pm 0.046(p<0.01)$ in HepG2 cells and $0.873 \pm 0.063$ vs. $0.586 \pm 0.034(p<0.01)$ in SNU449 cells before and after restoration of BCL6B expression (Figure 3B). It indicates that cell viability was suppressed by BCL6B in HCC cells. To explore the effect of BCL6B on apoptosis, flow cytometry was employed. Early apoptosis was detected by staining phosphatidylserin (PS) with Annexin V. The ratio of apoptosis was $1.06 \pm 0.72 \%$ vs. $4.73 \pm 0.21 \%$ in HepG2 cells $(p<0.01)$, and $2.06 \pm 0.90 \%$ vs. $7.8 \pm 0.95 \%$ in SNU449 cells $(p<0.01)$ before and after re-expression of BCL6B. Late apoptosis was evaluated by propidium iodide (PI) staining for broken down DNA. The ratio of apoptosis was $1.80 \pm 0.80 \%$ vs. $4.73 \pm 1.75 \%$ in HepG2 cells $(p>0.05)$ and $0.86 \pm 0.61 \%$ vs. $0.97 \pm 0.81 \%$ in SNU449 cells $(p>0.05)$ before and after re-expression of BCL6B (Figure 3C). Above results suggest that BCL6B induces apoptosis in the early step of liver carcinogenesis.

The effect of BCL6B on cell cycle was evaluated by flow cytometry. The cell phase distribution in HepG2 cells before and after re-expression of BCL6B was as follow: G1 phase: $60.32 \pm 0.31 \%$ vs. $71.73 \pm 1.71 \%(p<0.01)$, S phase: $27.40 \pm 0.71 \%$ vs. $18.53 \pm 1.39 \%(p<0.01), \mathrm{G} 2 / \mathrm{M}$ phase: $12.26 \pm 1.02 \%$ vs. $9.74 \pm 1.39 \%(p<0.01)$. The cell phase distribution in SNU449 cells before and after re-expression of BCL6B was as follow: G1 phase: $56.14 \pm 1.03 \%$ vs. $70.90 \pm 0.92 \%(p<0.01)$, $\mathrm{S}$ phase: $28.10 \pm 1.32 \%$ vs. 21.36 $\pm 1.38 \%(p<0.05), \mathrm{G} 2 / \mathrm{M}$ phase: $15.77 \pm 1.60 \%$ vs. 7.74 $\pm 0.48 \%(p<0.01)$. These results indicate that $\mathrm{G} 1 / \mathrm{S}$ arrest was induced by BCL6B (Figure 3D) in HCC cells.

\section{EGR1 was up-regulated by BCL6B in HCC cells}

To understand the mechanism of BCL6B on HCC carcinogenesis, gene expression microarray was employed in this study. As shown in Figure 4A, 
A

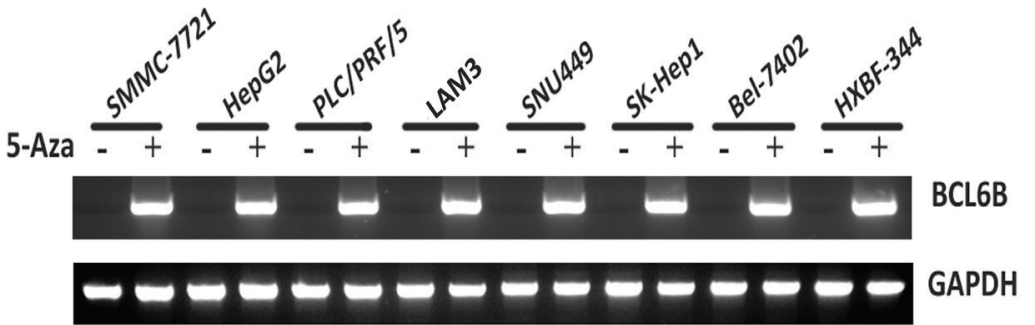

B
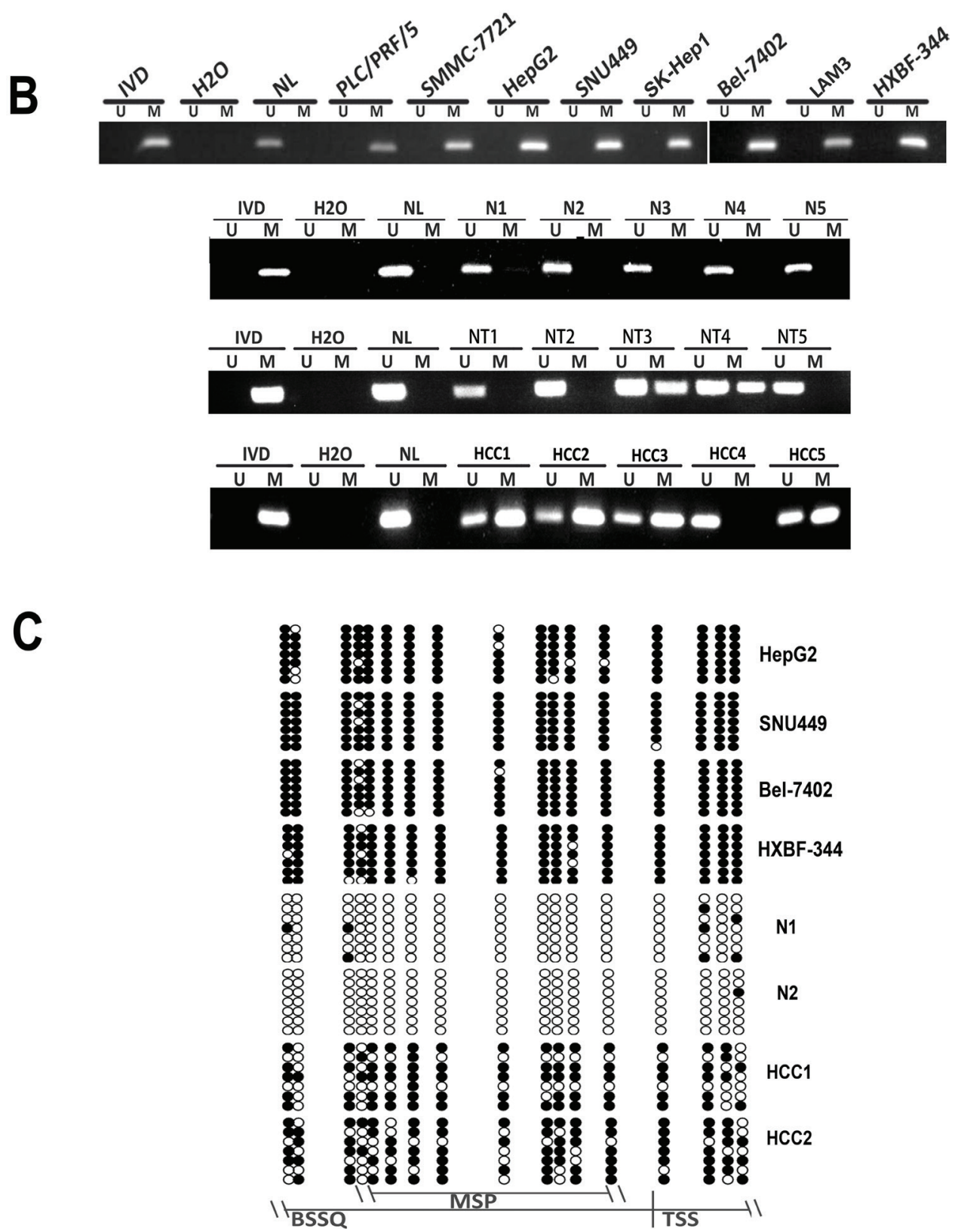

Figure 1: BCL6B expression was silenced by promoter region hypermethylation in HCC. A. The expression of BCL6B was detected by semi-quantitative RT-PCR before and after 5-Aza treatment in HCC cell lines. GAPDH: internal control. (+): 5-Aza treated, (-):5Aza untreated; SMMC-7721, HepG2, PLC/PRF5, LAM3, SNU449, Sk-hep1, Bel-7402 and HBXF344 are HCC cell lines B. Methylation specific PCR results of BCL6B gene in human HCC. U: unmethylated allele; M: methylated allele; IVD: in vitro methylated DNA; NL: normal lymphocyte DNA; NI, N2, N3, N4, N5: non-cancerous liver tissue samples: NT1, NT2, NT3, NT4 NT5: non-cancerouse tissue; HCC1, HCC2, HCC3, HCC4, HCC5: primary HCC samples. C. BSSQ results of BCL6B promoter region in HepG2, SNU449, HXBF344, BeL-7402, N1, N2, HCC1, HCC2. MSP: MSP amplification region; Filled circle: methylated CpG sites; opened circle: unmethylated CpG sites; TSS: transcription start site; BSSQ: BSSQ amplification region. 
Table 1: Univariate analysis of relationship between BCL6B methylation and clinicopathologic features of HCC patients

\begin{tabular}{|c|c|c|c|c|c|}
\hline \multicolumn{6}{|c|}{ BCL6B methylation status } \\
\hline Variable & No. & Methylated $(N=129)$ & Unmethylated $(N=20)$ & $\chi^{2}$ & $P$ value \\
\hline Gender & & & & 3.47 & 0.06 \\
\hline Male & 88 & 80 & 8 & & \\
\hline Female & 61 & 49 & 12 & & \\
\hline Age (years) & & & & 0.01 & 0.91 \\
\hline$\leq 65$ & 114 & 99 & 15 & & \\
\hline$>65$ & 35 & 30 & 5 & & \\
\hline Size (cm) & & & & 3.78 & 0.15 \\
\hline$\leq 3$ & 67 & 59 & 8 & & \\
\hline $3-5$ & 47 & 43 & 4 & & \\
\hline$>5$ & 35 & 27 & 8 & & \\
\hline Differentiation & & & & 0.02 & 0.99 \\
\hline High & 39 & 34 & 5 & & \\
\hline Middle & 80 & 69 & 11 & & \\
\hline Low & 30 & 26 & 4 & & \\
\hline Type of hepatisis & & & & 9.14 & $0.03 *$ \\
\hline $\mathrm{HBV}$ & 130 & 114 & 16 & & \\
\hline $\mathrm{HCV}$ & 9 & 5 & 4 & & \\
\hline $\mathrm{HBV}+\mathrm{HCV}$ & 1 & 1 & 0 & & \\
\hline $\mathrm{N}$ & 9 & 9 & 0 & & \\
\hline TNM & & & & 4.56 & 0.10 \\
\hline I & 90 & 79 & 11 & & \\
\hline II & 24 & 23 & 1 & & \\
\hline III & 35 & 27 & 8 & & \\
\hline
\end{tabular}

${ }^{a} P$ values are obtained from chi-square test.

${ }^{*}$ Statistically significant, $p<0.05$.

167 genes were up-regulated and 63 genes were downregulated over 3 times after re-expression of BCL6B in SNU449 cells. Among these genes, HMGA2, CLDN1, TFPI2, KIAA0101 and EGR1 are cancer related genes according to Diseases Association Analysis (http://bioinfo .vanderbilt.edu/webgestalt/). HMGA2, CLDN1, TFPI2 and EGR1 are up-regulated for 5, 3.3, 4.8 and 31.4 times, and KIAA0101 was down-regulated for 3.2 respectively (Figure 4B). Even through, the tendency of expression changes of these genes, which detected by quantitative RTPCR, was similar with microarray analysis (Supplementary Figure S1). Apparent increasing expression was only found in EGR1 gene by semi-quantitative RT-PCR and western blot in BCL6B re-expressed HepG2 and SNU449 cells (Figure 4C).

\section{Restoration of BCL6B expression activates p53 signaling}

EGR1 is a component of p53 signaling, which suppresses cell growth in human lung cancer [13-17]. To analyze the effect of BCL6B in p53 signaling in HepG2 and SNU449 cells, first we eliminated the possible effect of p53 mutation on these cells. No p53 mutation was found in HepG2 cells. While SNU449 carries a G to A substitution at nucleotide 481 of the p53 gene, leading to 
A

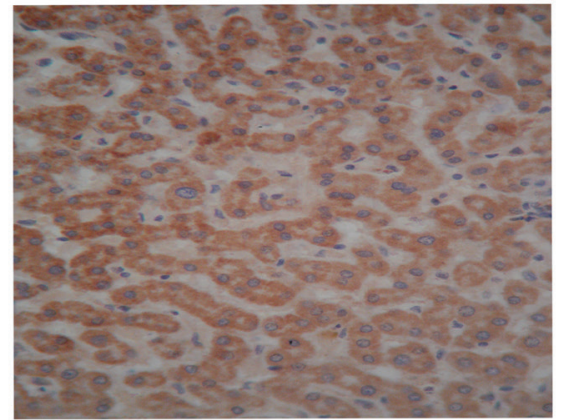

Adjacent

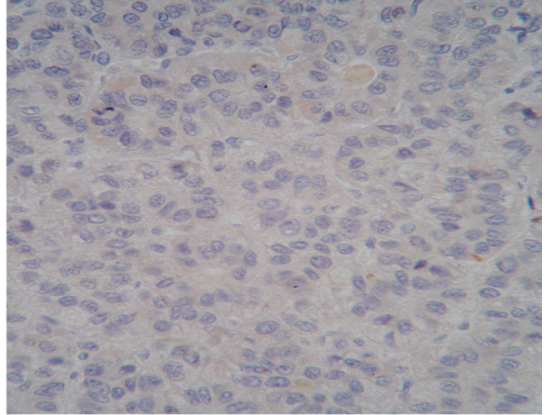

Cancer

B

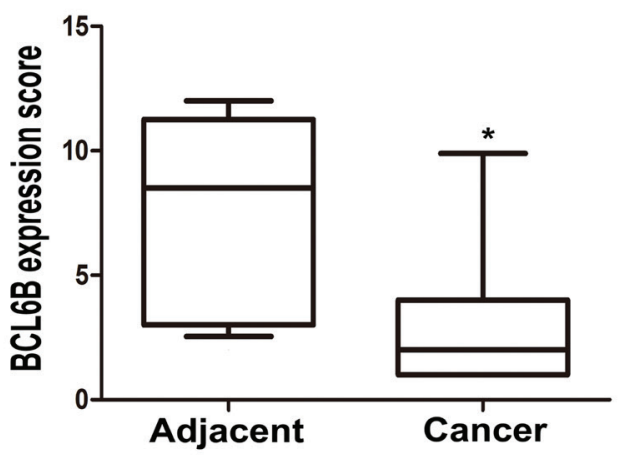

C

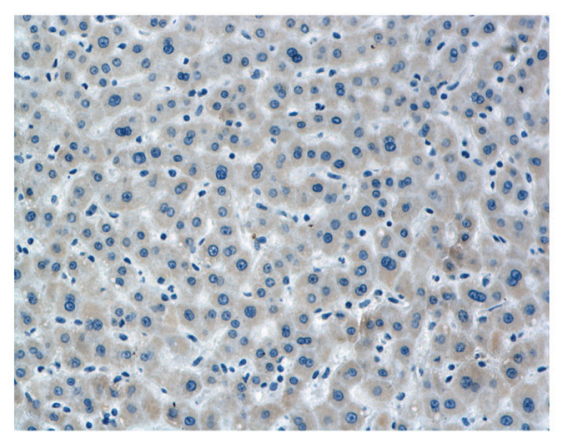

Methylated HCC

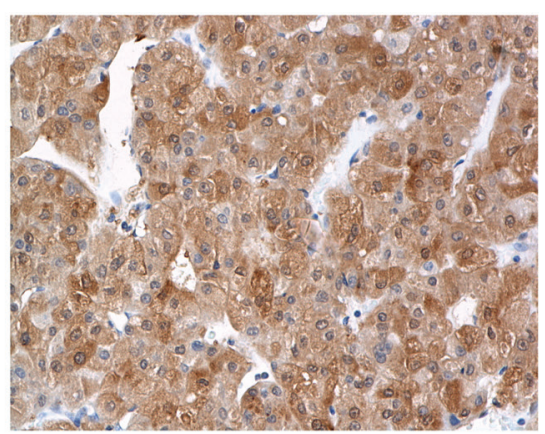

Unmethylated HCC

D

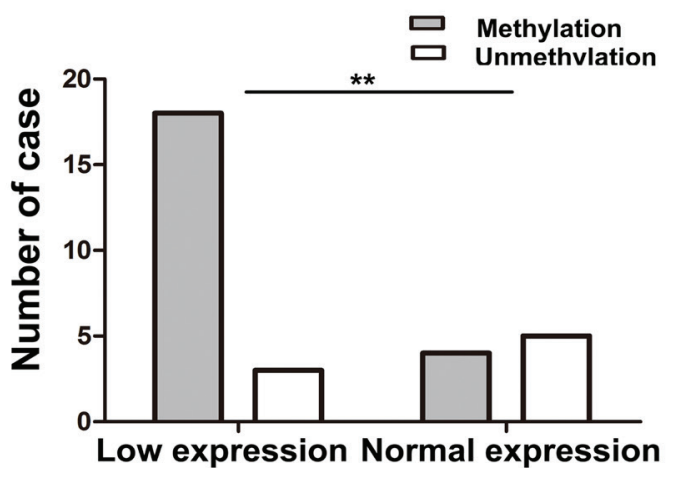

Figure 2: Reduced expression of BCL6B was associated with promoter region hypermethylation in human primary HCC. A. Representative BCL6B staining in matched human primary HCC (right) and adjacent tissue samples (left) by IHC. (X200). B. The scores of BCL6B expression in 30 matched HCC and adjacent tissue samples are shown as box plots. Horizontal lines in the boxes represent the median score; the bottom and top lines of the boxes represent the 25th and 75th percentiles, respectively; vertical bars represent the range of data. $\left({ }^{*} p<0.05,{ }^{* *} p<0.01\right)$ C. Representative BCL6B staining in methylated (left) and unmethylated HCC (right) by IHC. (X200) D. Loss of/reduced expression of BCL6B was associated with promoter region hypermethylation in human HCC. $(* p<0.05$, $* * p<0.01)$ 

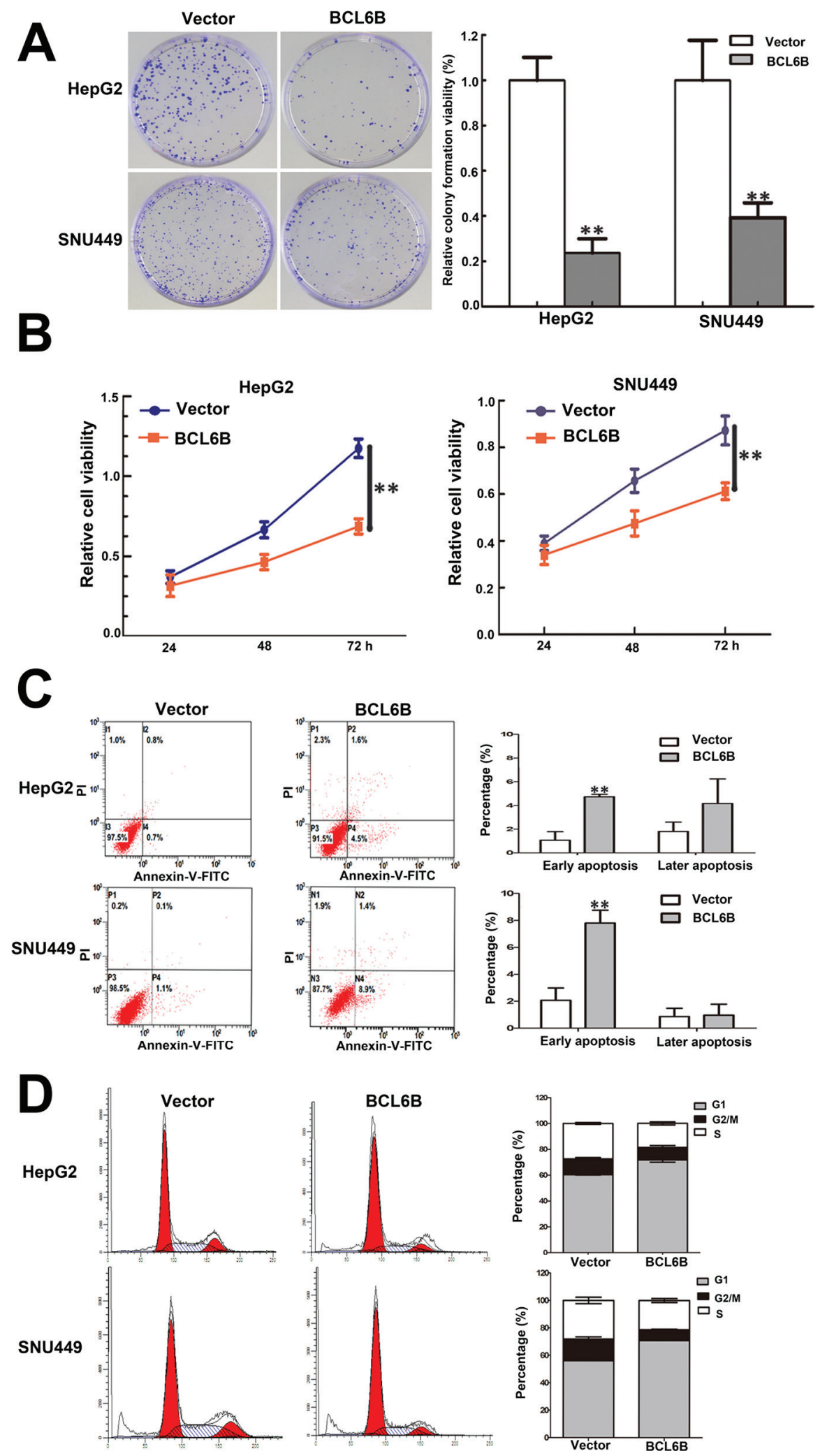

Figure 3: Restoration of BCL6B expression inhibited proliferation, induced aopotosis and G1/S arrest in HCC cells. A. Colony formation in BCL6B re-expressed and unexpressed HepG2 and SNU449 cells. Each experiment was repeated for three times. ${ }^{*} p<0.01$. B. Growth curves of BCL6B re-expressed and unexpressed HepG2 and SNU449 cells analyzed by CCK-8 assay. Each experiment was repeated for three times. ${ }^{* *} p<0.01$. C. Representative apoptosis data analyzed by flow cytometry in BCL6B re-expressed and unexpressed HepG2 and SNU449 cells. Each experiment was repeated for three times. ** $p<0.01$. D. Cell cycle distribution in BCL6B re-expressed and unexpressed HepG2 and SNU449 cells. Each experiment was repeated for three times. 


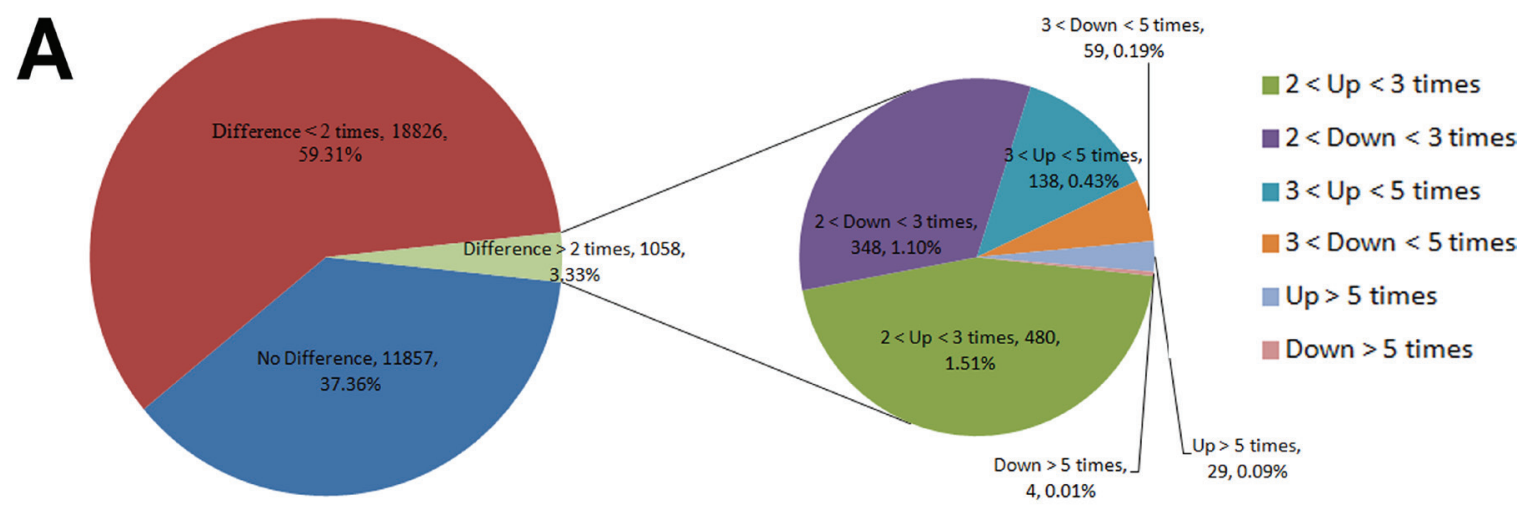

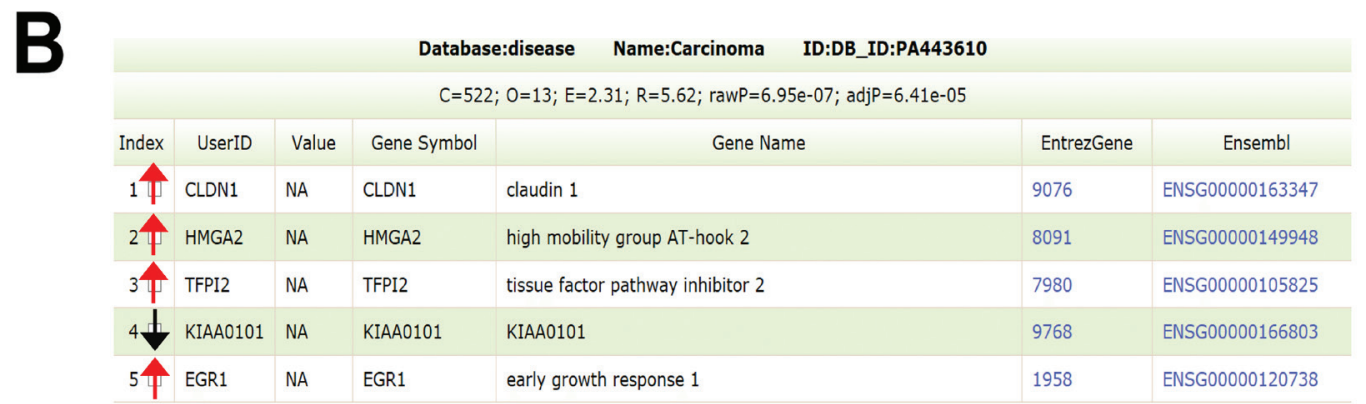
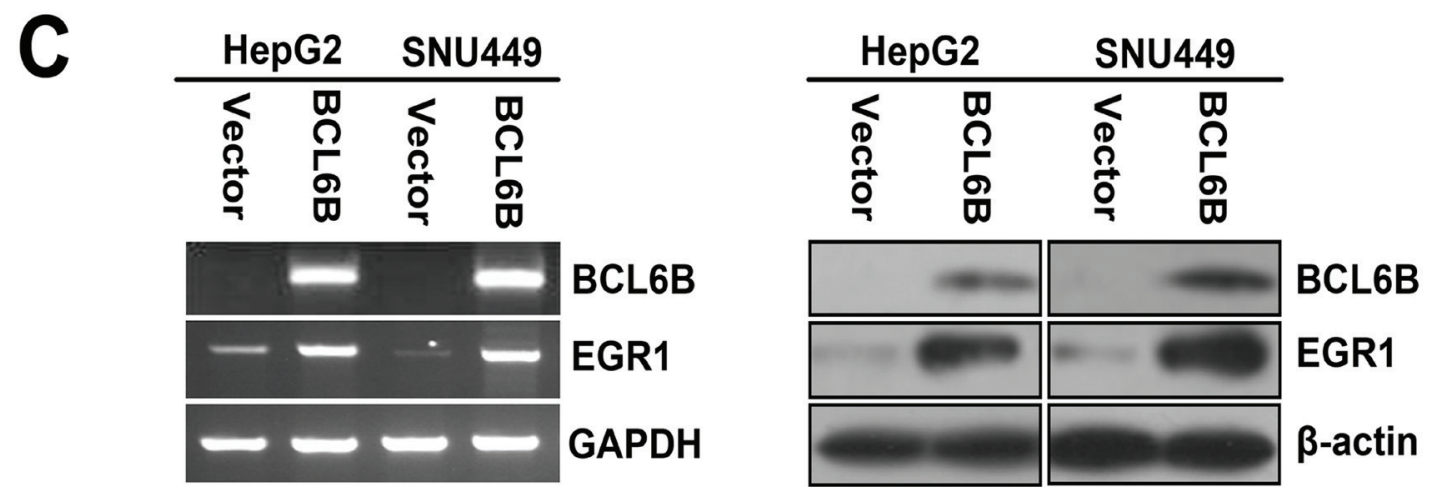

Figure 4: Differentially expressed genes in BCL6B re-expressed and unexpressed SNU449 cells analyzed by gene expression array. A. The pie chart analysis for all 31741 probes in BCL6B re-expressed and unexpressed SNU449 cells. 167 genes were up regulated and 63 genes were down regulated for more than 3 times. B. HMGA2, CLDN1, TFPI2, KIAA0101 and EGR1 are cancer related genes according to Diseases Association Analysis. HMGA2, CLDN1, TFPI2 and EGR1 which are up-regulated for 5, $3.3,3.7$ and 31.4 times, and KIAA0101 is down-regulated for 3.2 times, respectively. (Red arrow stands for up-regulated and black arrow stands for down-regulated genes) C. The expression of EGR1 was validated by semi-quantitative RT-PCR and Western Blot in BCL6B unexpressed and re-expressed HepG2 and SNU449 cells.

an alanine to threonine mutation at codon 161 [18]. But this mutation site is not related to the interacting region of 553 with EGR1 [19]. To further understand the role of BCL6B in HCC, the expression of EGR1 and p53 were detected before and after re-expression of BCL6B in HepG2 and SNU449 cells. The expression of EGR1 and p53 were increased apparently after restoration of BCL6B expression in HepG2 and SNU449 cells. As shown in Figure 5A \& 5B, the expression of p21, caspase-3, caspase-7, caspase-9, PARP and Bax were increased and the expression of MDM2 and bcl-2 were reduced in BCL6B expressed HepG2 and SNU449 cells. These results suggest that $\mathrm{p} 53$ signaling was activated by BCL6B through EGR1. And BCL6B induce apoptosis in HCC cells.

\section{P53 signaling was inhibited by knocking down BCL6B or EGR1 in DKO cells}

To validate $\mathrm{p} 53$ signaling was activated by BCL6B via EGR1, BCL6B and EGR1expressed DKO cells was employed. As shown in Figure 6, the expression of 


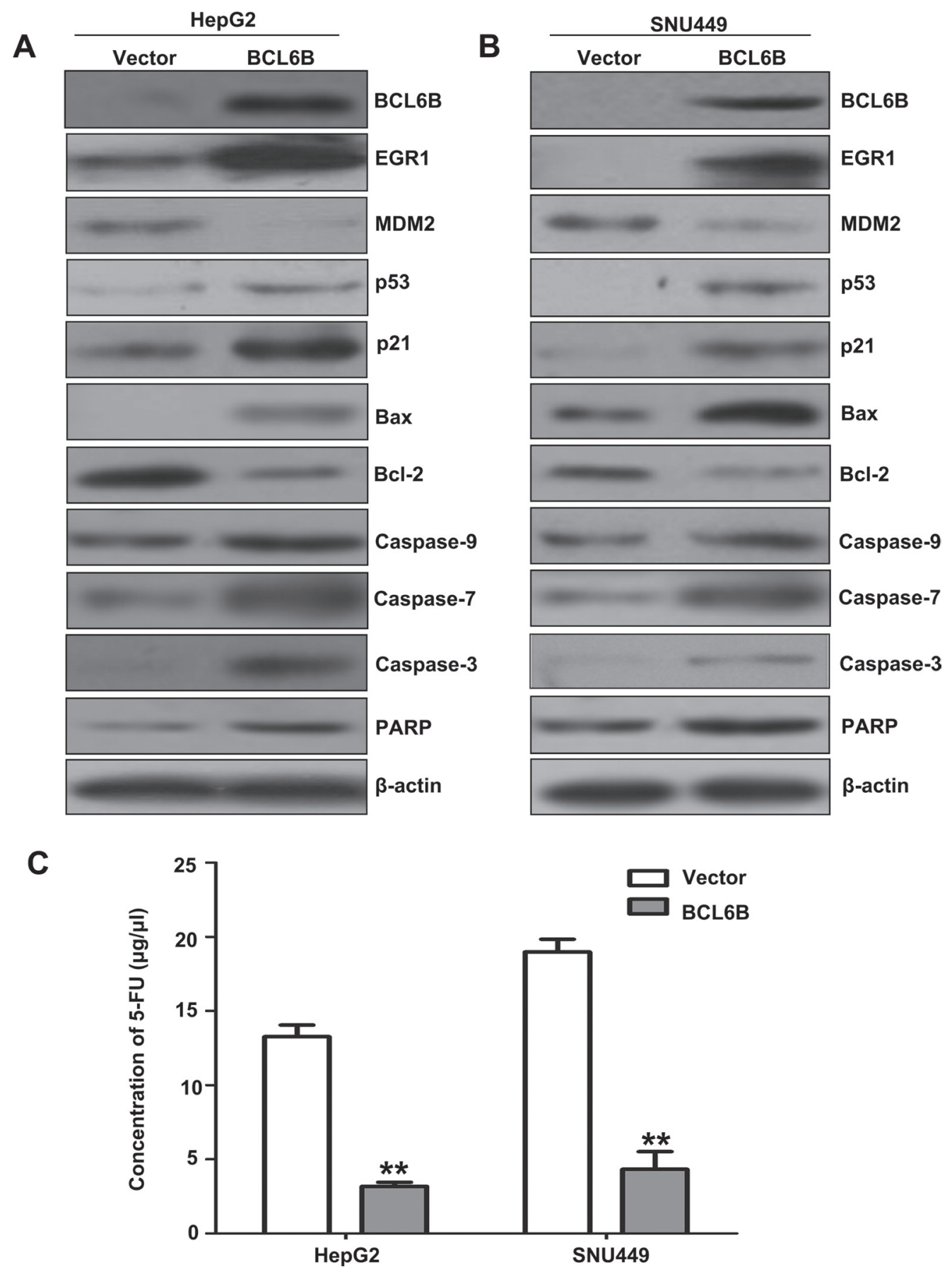

Figure 5: The effect of BCL6B on p53 signaling and apoptosis in HCC cells. A\&B. The expression of BCL6B, EGR1, MDM2, p53, p21, Bax, bcl-2, caspase-9, caspase-7, caspase-3 and PARP was detected by western blot in BCL6B unexpressed and re-expressed HepG2 and SNU449 cells. $\beta$-actin: internal control. C. IC50 value of 5-FU in BCL6B unexpressed and re-expressed HepG2 and SNU449 cells. The IC50 value of 5-FU was evaluated by CCK-8 activity. White columns: the IC50 value of 5-FU in BCL6B unexpressed HepG2 and SNU449 cells. Gray columns: the IC50 value of 5-FU in BCL6B re-expressed HepG2 and SNU449 cells.

EGR1, p53, p21, Bax, caspase-3, caspase-7, caspase-9 and PARP were reduced and MDM2 and bcl-2 were increased by knocking down BCL6B (Figure 6A). Similar to BCL6B knocking down, the expression of p53, p21, Bax, caspase-3, caspase-7, caspase-9 and PARP were reduced and MDM2 and bcl-2 were increased after knock down EGR1, while no change was found for BCL6B expression (Figure 6B). These results indicate $\mathrm{p} 53$ signaling was activated by $\mathrm{BCL} 6 \mathrm{~B}$ via EGR1. 
A
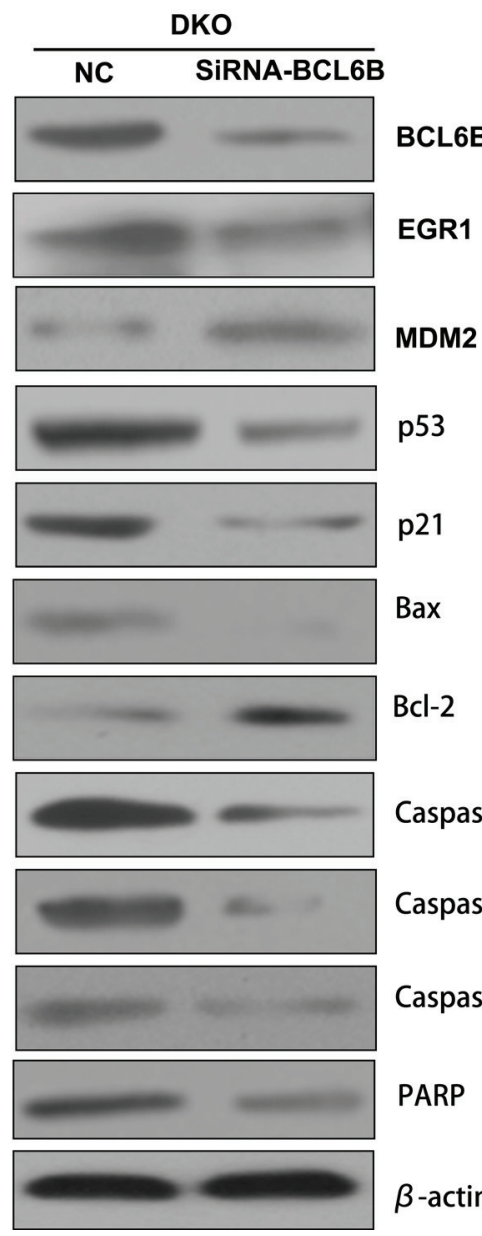

BCl-2

PARP

\section{B}

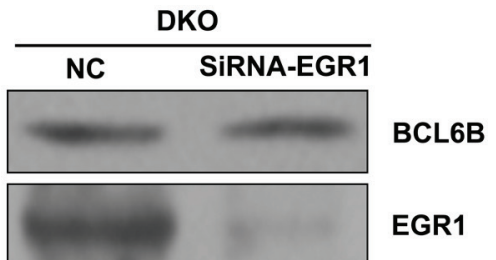

Caspase 9

Caspase 7

Caspase 3

$\beta$-actin

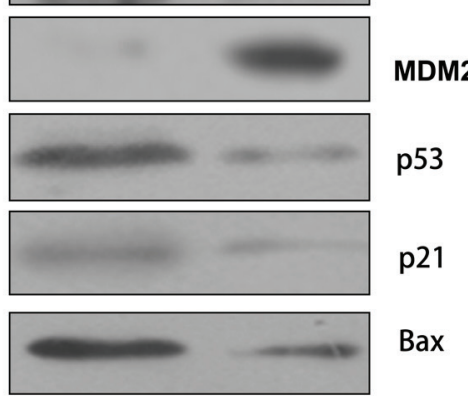

$\mathrm{BCl}-2$
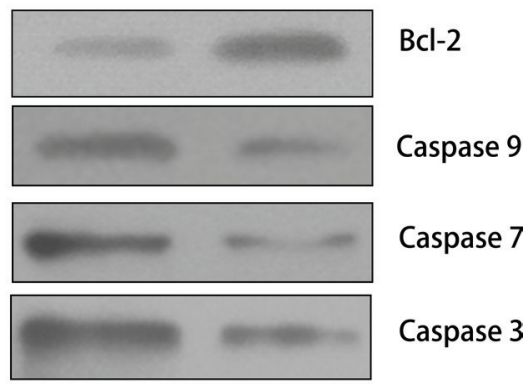

Caspase 3

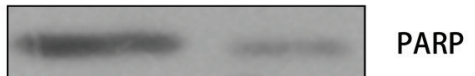

PARP

$\beta$-actin

Figure 6: P53 signaling was activated by BCL6B through EGR1 in DKO cell line. A. The expression of BCL6B, EGR1, MDM2, p53, p21, Bax, bcl-2, caspase-9, caspase-7, caspase-3 and PARP was detected by western blot in BCL6B expressed and siRNA knocking down DKO cell line. $\beta$-actin: internal control. B. The expression of BCL6B, EGR1, MDM2, p53, p21, Bax, bcl-2, caspase-9, caspase-7, caspase-3 and PARP was detected by western blot in EGR1 expressed and siRNA knocking down DKO cell line. $\beta$-actin: internal control.

\section{Restoration of BCL6B expression sensitized HCC cells to 5-FU}

The tumor suppressor p53 maintains DNA integrity by transcriptionally activating genes such as p21 (CDKN1N) and GADD45a, the products of which induce cell-cycle arrest in response to DNA damage [20-22]. However, depending on the cellular context and the nature of the DNA damage, p53 can trigger elimination of the damage cells by promoting apoptosis through the induction of pro-apoptosis genes, such as Bax, and the down-regulation of anti-apoptosis $\mathrm{Bcl} 2[23,24]$. In vitro studies have reported that loss of $\mathrm{p} 53$ function reduces cellular sensitivity to 5-FU [25, 26]. Disrupting both alleles of TP53 or Bax in colon cancer cell line made the cells strikingly resistant to apoptosis induced by $5-\mathrm{FU}$ compared with the parental line $[25,27,28]$. 5-FU is widely used for cancer treatment and still the mainstay of chemotherapy. Apoptosis was induced by activating p53 signaling after restoration of BCL6B expression in HepG2 and SNU449 cells. To see if BCL6B is involved in 5-FU sensitivity in HCC cells, the sensitivity of HepG2 and SNU449 cells to 5-FU was analyzed before and after re-expression of BCL6B. The IC50 of 5-FU was $13.27 \pm 0.77$ vs. $3.18 \pm 0.28 \mu \mathrm{g} / \mathrm{ml}(p<0.01)$ in HepG2 and $18.98 \pm 0.86$ versus $4.33 \pm 1.18 \mu \mathrm{g} / \mathrm{ml}(p<0.01)$ in SNU449 cells before and after restoration of BCL6B expression (Figure 5C). The results suggest that BCL6B sensitized HCC cells to 5-FU.

\section{DISCUSSION}

BCL6B is frequently methylated in human hepatocellular carcinoma and the expression of BCL6B was regulated by promoter region hypermethylation. It suggests that BCL6B is a potential HCC detection marker and BCL6B methylation may involved in hepatocellular 
carcinogenesis. Increasing evidence reveals that viral genes are one of the key players in regulating DNA methylation. Particularly in cancers closely associated with the hepatitis B virus, simian virus 40 (SV40), and Epstein-Barr virus [29]. In vitro, $\mathrm{HBx}$ has been documented to disturb the host DNA methylation system by up-regulating DNMT expression and activities, which in turn results in hypermethylation and repression of some tumor suppressor genes (insulin-like growth factor binding protein-3 gene, E-cadherin), thus increasing tumor susceptibility [29-31]. Previous studies have shown that HCC is associated with hypermethylation of several tumor suppressor genes [32, 33]. We and other groups have shown that some genes are preferentially methylated in HCV-related HCC $[6,34]$. In this study, we found that BCL6B methylation is related to $\mathrm{HBV}$ positive $\mathrm{HCC}$. The result further suggests that HBV may promote hepatocellular carcinogenesis by inducing promoter region methylation in tumor suppressor. Further study found that BCL6B suppresses cell proliferation and induces apoptosis and G1/S arrest in HCC cells. To further understand the mechanism of BCL6B on hepatocellular carcinogenesis, gene expression microarray was employed. By analyzing the expression changes of genes before and after re-expression of BCL6B in methylated HCC cell lines, 167 genes were up-regulated and 63 genes were downregulated over 3 times after re-expression of BCL6B in SNU449 cells. Among these genes, HMGA2, CLDN1, TFPI2, KIAA0101 and EGR1 are cancer related genes according to Diseases Association Analysis and increased expression of EGR1 was confirmed by further semiquantitative RT-PCR, quantitative RT-PCR and western blot in BCL6B re-expressed HepG2 and SNU449 cells. EGR1 was reported to suppress cell growth by regulating p53 in lung cancer cells and BCL6B was reported involved in p53 signaling in gastric cancer [11, 15-17]. Thus, we focused on the p53 signaling pathway in human HCC. The expression of EGR1, p53, p21, caspase-3, caspase-7, caspase-9, PARP and Bax was increased and the expression of MDM2 and bcl-2 was reduced after restoration of BCL6B expression in human HCC cells. These results suggest that BCL6B activated p53 signaling and induced apoptosis in human HCC cells. As p53 expression was regulated by EGR1 in human lung cancer cells $[15,17]$. It is reasonable to suggest that p53 signaling was activated by BCL6B through EGR1 in human HCC. It was reported before that p53 disruption rendered cells strikingly resistant to the effects of the antimetabolite $5-\mathrm{FU}$ in colorectal cancer [25]. We further analyzed the sensitivity of BCL6B re-expressed and unexpressed HCC cells to 5-FU. The results demonstrated that BCL6B sensitized HCC cells to 5-FU. These results indicate that BCL6B sensitizes HCC cell to 5-FU by activating p53 signaling.

In conclusion, BCL6B is frequently methylated in human $\mathrm{HCC}$ and the expression of BCL6B was regulated by promoter region hypermethylation. BCL6B suppresses cell proliferation and induces apoptosis in HCC cells. EGR1, a key component of p53 signaling, was up regulated by BCL6B in HCC cells. BCL6B activates p53 signaling and sensitizes $\mathrm{HCC}$ cells to 5-FU.

\section{MATERIALS AND METHODS}

\section{Primary human hepatic cancer samples and cell lines}

One hundred and forty-nine cases of primary hepatic cancer were collected as fresh frozen tissue from Chinese PLA General Hospital. All cases of hepatic cancer were classified by TNM stage, including stage I $(N=90)$, stage II $(N=24)$ and stage III $(N=35)$. Eight cases of normal live tissue were collected as fresh frozen tissue from hepatic hemangioma patients and 50 cases of adjacent tissue samples were from primary hepatic cancer patients in Chinese PLA General Hospital. Among 149 cancer samples, 30 cases of paraffin blocks are available with matched adjacent tissue. All samples were collected under the approved guidelines of the Chinese PLA General Hospital's institutional review board.

Nineteen human hepatic cancer cell lines HepG2, SNU449, PLC/PRF/5, SK-Hep1, SMMC-7721, HBXF344, LAM3, SNU182, SNU387, SNU475, QSG7701, QGY-7703, BEL-7404, BEL-7405, MHCC97H, Huh1, Huh7, Hep3B and Bel-7402, and DKO cells (double knockouting of DNMT1 and DNMT3b in HCT116 cell) were included in this study [35]. All of HCC cell lines were previously established from primary hepatocellular carcinoma. Each of the cell lines were maintained in 90\% RPMI 1640 (Intrivogen, Carlsbad, CA, USA), supplemented with 10\% fetal bovine serum (Hyclone, Logan, UT). Cells were passaged $1: 3$ once total confluence (approximately $10^{6}$ cells) was reached on a $75 \mathrm{~cm}^{2}$ culture flask (NEST Biotechnology, Jiangsu, China).

\section{5-aza-2'-deoxycytidine (5-Aza) treatment}

HCC cell lines were split to low density (30\% confluence) 12 hours before treatment. Cells were treated with 5-aza-2'-deoxy -cytidine (5-Aza) (Sigma, St. Louis, MO, USA) at a concentration of $2 \mu \mathrm{M}$. Growth medium, conditioned with DAC at $2 \mathrm{uM}$, was exchanged every $24 \mathrm{~h}$ for total $96 \mathrm{~h}$ treatment.

\section{RNA isolation and semi-quantitative reverse transcription PCR}

Total RNA was isolated using Trizol reagent (Life Technologies, Gaithersburg, MD) and chloroform, isopropanol precipitation. Agarose gel (1\%) electrophoresis and spectrophotometric analysis 
(A260:280 nm ratio) was used to evaluate RNA quality and quantity. Reverse transcription of RNA $(5 \mu \mathrm{g})$ was carried out according to the instruction of Superscript III-reverse transcriptase kit (Invitrogen, Carlsbad, CA). Following first strand synthesis, the cDNA was diluted to $100 \mu \mathrm{l}$ using water. Subsequently, $2.5 \mu 1$ of the diluted cDNA mixture was used for PCR amplification in a final volume of $25 \mu \mathrm{l}$. A total of 35 cycles was amplified. GAPDH was amplified with 25 cycles to ensure cDNA quality and quantity for each RT-PCR. RT-PCR primers of BCL6B are as follow: Sense: 5'- AAGCCGTATAAGTGTGAGACG -3', antisense: 5'- CCGAGAATGTGGTAGTGCAC -3'. Amplified products were analyzed on $2 \%$ agarose gels.

\section{DNA extraction and bisulfite modification}

HCC cells and tissue samples were digested with proteinase $\mathrm{K}$. Then genomic DNA was isolated by phenolchloroform extraction. The extracted DNA was diluted in TE buffer and stored at $-20^{\circ} \mathrm{C} .2 \mu \mathrm{g}$ of genomic DNA was diluted in $50 \mu \mathrm{l}$ of water. The bisulfite treatment was carried out for $16 \mathrm{~h}$ at $50^{\circ} \mathrm{C}$. DNA samples were then purified with the Wizard DNA Clean-Up System (Promega, WI, USA), desulfonated with $\mathrm{NaOH}$, and then precipitated with ethanol and resuspended in $20 \mu \mathrm{l}$ of water.

\section{Methylation-specific PCR (MSP) and bisulfite sequencing (BSSQ)}

MSP and BSSQ were performed as described previously [36, 37]. Each PCR assay included a methylation control, an unmethylation control and water control. MSP and BSSQ primers were designed around the transcriptional start site in the promoter region of BCL6B (NM_181844.3). The primers sequences are as follow: unmethylation-forward: 5'-TTTTGTTTTGGATTTGTTATTTGGAGAGT-3', unmethylation-reverse:

5'-CTTAACCTCAACTCCTTTATCTAACCA-3', methylation-forward:

5'- CGTTTTGGATTCGTTATTTGGAGAGC-3', methylation-reverse:

5'- TAACCTCGACTCCTTTATCTAACCG -3'. BSSQforward: 5'- GTTTTTGAGGTTTYGTTTTYGAG -3' BSSQ-reverse: 5'- CCTCAATCTCTTATTCTTACCC -3'. The spanning of MSP and BSSQ amplification region of BCL6B are 119bp and 227bp, respectively.

\section{Immunohistochemistry}

Immunohistochemical staining for BCL6B was performed on $4 \mu \mathrm{m}$ thick serial sections derived from 30 cases available matched cancer and adjacent tissue paraffin blocks using antibody against BCL6B (1:100 dilution, Abcam, Cambridge, Massachusetts, USA) as described previously $[38,39]$. The staining intensity and area extent were graded according to the German semiquantitative scoring system, which has been widely accepted and used in previous studies [40-42]. In brief, staining intensity of the nucleus, cytoplasm, and/or membrane (no staining $=0$; weak staining $=1$; moderate staining $=2$; strong staining $=3$ ); extent of stained cells $(0 \%=0,1-24 \%=1,25-49 \%=2,50-74 \%=3,75-$ $100 \%=4)$. The final immunoreactive score ( 0 to 12$)$ was determined by multiplying intensity scores with the extent of positivity scores of stained cells. The cutoff value is 4 .

\section{Colony formation and cell viability assay}

Cells were plated in 6-well culture plates $24 \mathrm{~h}$ before transfection and then were transfected with empty vector pcDNA3.1 or the pcDNA3.1-HA-BCL6B vector. The transfected cells were diluted and reseeded $24 \mathrm{~h}$ later. Cells were seeded at 1000 cells and 1, 500 cells per well in six-well culture plates in triplicate for HepG2 and SNU449 cells. Transfected cells were selected in $400 \mathrm{ug} / \mathrm{mL}$ G418 (Invitrogen). After 14 days, colonies were fixed with $75 \%$ ethanol for $30 \mathrm{~min}$ and stained with $0.2 \%$ crystal violet for visualization and counting. The number of colonies was counted under the microscope.

Cells $\left(5 \times 10^{3}\right)$ were seeded in 96 well plates, incubated for 24 hours and transfected with pcDNA3.1HA-BCL6B or the empty vector pcDNA3.1. Cell viability was determined by the 3-(4, 5-dimethylthiazol2-yl) -5-(3-carboxymethoxyphenyl)-2-(4-sulfophenyl )-2 H-tetrazolium (MTS) assay (Promega, Madison, Wisconsin, USA) at $24 \mathrm{~h}, 48 \mathrm{~h}$ and $72 \mathrm{~h}$ after treatment, respectively. Absorbance was measured on a microplate reader (Thermo Multiskan MK3 USA) at a wavelength of $490 \mathrm{~nm}$. The results were plotted as means $\pm \mathrm{SD}$. All assays were performed in triplicate and repeated for three times. The percentage of viable cells $(\%)=\left[\mathrm{A}_{450-630}(\right.$ treated $)$ $\mathrm{A}_{450-630}$ (blank)]/[ $\mathrm{A}_{450-630}$ (control) - $\mathrm{A}_{450-630}($ blank $\left.)\right] \times 100 \%$. $\mathrm{IC}_{50}$ was defined as the concentration, which was required for $50 \%$ inhibition of cell growth. The values were calculated by nonlinear regression analysis using SPSS 17.0 software (SPSS Inc., Chicago, IL).

\section{Flow cytometry analysis}

For cell cycle analysis, briefly, after 12 hours of synchronization by serum starvation, the HepG2 and SNU449 cells transfected with BCL6B expression vector or control empty vector 24 hours before were re-stimulated with $10 \%$ fetal bovine serum (FBS) for 24 hours. Cells were fixed in $70 \%$ ethanol and stained with $50 \mu \mathrm{g} / \mathrm{ml}$ propidium iodide (BD Pharmingen, San Jose, CA). The cells were then sorted by FACS Calibur (BD Biosciences, Franklin Lakes, NJ) and cell-cycle profiles were analyzed by ModFit version 2.0 (Verity Software House, Topsham, ME) as described in the previous article [7, 39]. 
Histograms were analyzed for cell cycle compartments using Early and late apoptosis was detected by Annexin V- FITC/propidium iodide (PI) Apoptosis Detection Kit (KeyGen Biotechnology, China).

\section{In vitro chemosensitivity testing for $\mathrm{HCC}$ cells}

Cells were seeded in 96-well plates and treated with 5 -FU at the dose of $0,0.36,0.73,1.56,3.12,6.25$, $12.5,25,50,100 \mu \mathrm{g} / \mu \mathrm{l}$. The percentage of viable cells was evaluated as described above. IC50 was defined as the concentration, which was required for $50 \%$ inhibition of cell growth.

\section{Gene expression microarray analysis}

RNA from BCL6B expressed and unexpressed SNU449 cells were converted to cDNA labeled with fluorescent dye (Cy3). The labeled cDNA were mixed and hybridized to human gene expression array and then washed, scanned, and analyzed. The gene expression array (Agilent Human $(4 \times 44 \mathrm{k}))$ was provided by Agilent Technologies. The ratio represents gene expression alterations between BCL6B expressed and unexpressed groups. The expression changed more than 3 -fold was further analyzed by the software of Diseases Association Analysis (http://bioinfo.vanderbilt.edu/ webgestalt/) and validated by semi-quantitative RT-PCR and western blot.

\section{SiRNA knock down}

Selected siRNAs targeting BCL6B and EGR1 and RNAi negative control duplex were used in this study. The siRNA-BCL6B sequences are as follows: siRNA duplex (sense: 5'-GACGAAGACAAACCCUAUATT-3'; antisense: 5'-UAUAGGGUUUGUCUUCGUCTT -3'); The siRNA-EGR1 sequences are as follows: siRNA duplex (sense: 5'-GCAAGAGGCAUACCAAGAUTT-3'; antisense: 5'-AUCUUGGUAUGCCUCUUGCTT -3'); RNAi negative control duplex (sense: 5'-UUCUCCGAACGUGUCACGUT T-3'; antisense: 5'-ACGUGACACGUUCGGAGAAT T-3'). RNAi oligonucleotide or RNAi negative control duplex (GenePharma Co.) was transfected into DKO cells according to the manufacturer's instructions.

\section{Quantitative RT-PCR}

Quantitative RT-PCR was performed in triplicate with an Applied Biosystems Stepone Fast Sequence Detection System using TaqMan universal PCR master mix according to the manufacturer's protocol (Applied Biosystems). Levels of RNA expression were determined using the Stepone System software version 1.3.1 (Applied Biosystems).

\section{Protein preparation and western blot}

Protein preparation and western blot were performed according to our previous report [43]. The following primary antibodies were used: anti-BCL6B (Abcam, Cambridge, Massachusetts, USA), anti-EGR1 (Epitomics, MA, USA), anti-MDM2 (Abgent, San Diego, U.S.), anti-p53 (Bioworld Tech, MN, USA), anti-bax (Beyotime Biotech, China), anti-bcl-2 (Cell Signalinging Technology, USA), anti-caspase 3 (Abcam, MA, USA), anti-caspase 7 (Abcam, MA, USA), anticaspase 9 (Abcam, MA, USA) and cleaved PARP (Cell Signalinging Technology, USA), and $\beta$-actin (Beyotime Biotech, China). $\beta$-actin was used in a reprobing as a loading control. The blots were visualized using enhanced chemiluminescence (Pierce Bioscience, IL, USA).

\section{Statistical analysis}

Statistical analysis was performed using SPSS 17.0 software (SPSS Inc., Chicago, USA). Chi-square or Fischer's exact tests were used for evaluating the relationship between methylation status and clinic-pathological characteristics. All experiment data are presented as means \pm standard deviation (SD) of at least three independent experiments. Two-sided tests were used to determine significance, and $p<0.05$ was considered statistically significant.

\section{ACKNOWLEDGMENTS}

This work was supported by grants from the National Basic Research Program (973 Program No. 2012CB934002, 2010CB912802), National Key Scientific instrument Special Programme of China (Grant No. 2011YQ03013405), National High-tech R\&D Program (863 Program No. SS2012AA020314, SS2012AA020821, SS2012AA020303), National Science Foundation of China (Grant No. 81121004, No. 81071953, No. 81161120432, No. 81127006 and No. 81071210) and the International Cooperation Plan of National Science and Technology Department of China (No. 2012DFG32070).

\section{CONFLICTS OF INTEREST}

JGH is a consultant to MDxHealth. The other authors declare no conflict of interest.

\section{REFERENCES}

1. Siegel R, Naishadham D, Jemal A. Cancer statistics, 2013. CA Cancer J Clin. 2013; 63:11-30.

2. Jemal A, Bray F, Center MM, Ferlay J, Ward E, Forman D. Global cancer statistics. CA Cancer J Clin. 2011; 61:69-90. 
3. Faber W, Stockmann M, Schirmer C, Mollerarnd A, Denecke T, Bahra M, Klein F, Schott E, Neuhaus P, Seehofer D. Significant impact of patient age on outcome after liver resection for HCC in cirrhosis. Eur J Surg Oncol. 2014; 40:208-213.

4. Kawano Y, Sasaki A, Kai S, Endo Y, Iwaki K, Uchida H, Shibata K, Ohta M, Kitano S. Short- and long-term outcomes after hepatic resection for hepatocellular carcinoma with concomitant esophageal varices in patients with cirrhosis. Ann Surg Oncol. 2008; 15:1670-1676.

5. Feil R, Fraga MF. Epigenetics and the environment: emerging patterns and implications. Nat Rev Genet. 2011; 13:97-109.

6. Yang B, Guo M, Herman JG, Clark DP. Aberrant promoter methylation profiles of tumor suppressor genes in hepatocellular carcinoma. Am J Pathol. 2003; 163:1101-1107.

7. Zhu H, Wu K, Yan W, Hu L, Yuan J, Dong Y, Li Y, Jing K, Yang Y, Guo M. Epigenetic silencing of DACH1 induces loss of transforming growth factor-betal antiproliferative response in human hepatocellular carcinoma. Hepatology. 2013; 58:2012-2022.

8. Zhang X, Yang Y, Liu X, Herman JG, Brock MV, Licchesi JD, Yue W, Pei X, Guo M. Epigenetic regulation of the Wnt signaling inhibitor DACT2 in human hepatocellular carcinoma. Epigenetics. 2013; 8:373-382.

9. Jia Y, Yang Y, Liu S, Herman JG, Lu F, Guo M. SOX17 antagonizes WNT/beta-catenin signaling pathway in hepatocellular carcinoma. Epigenetics. 2010; 5:743-749.

10. Okabe S, Fukuda T, Ishibashi K, Kojima S, Okada S, Hatano M, Ebara M, Saisho H, Tokuhisa T. BAZF, a novel Bcl6 homolog, functions as a transcriptional repressor. Mol Cell Biol. 1998; 18:4235-4244.

11. Xu L, Li X, Chu ES, Zhao G, Go MY, Tao Q, Jin H, Zeng Z, Sung JJ, Yu J. Epigenetic inactivation of BCL6B, a novel functional tumour suppressor for gastric cancer, is associated with poor survival. Gut. 2012; 61:977-985.

12. Yang Q, Gao J, Xu L, Zeng Z, Sung JJ, Yu J. Promoter hypermethylation of BCL6B gene is a potential plasma DNA biomarker for gastric cancer. Biomarkers. 2013; 18:721-725.

13. Baron V, Adamson ED, Calogero A, Ragona G, Mercola D. The transcription factor Egrl is a direct regulator of multiple tumor suppressors including TGFbeta1, PTEN, p53, and fibronectin. Cancer Gene Ther. 2006; 13:115-124.

14. Liu J, Grogan L, Nau MM, Allegra CJ, Chu E, Wright JJ. Physical interaction between p53 and primary response gene Egr-1. Int J Oncol. 2001; 18:863-870.

15. Milbrandt J. A nerve growth factor-induced gene encodes a possible transcriptional regulatory factor. Science. 1987; 238:797-799.

16. Krones-Herzig A, Adamson E, Mercola D. Early growth response 1 protein, an upstream gatekeeper of the p53 tumor suppressor, controls replicative senescence. Proc Natl Acad Sci U S A. 2003; 100:3233-3238.

17. Krones-Herzig A, Mittal S, Yule K, Liang H, English C, Urcis R, Soni T, Adamson ED, Mercola D. Early growth response 1 acts as a tumor suppressor in vivo and in vitro via regulation of p53. Cancer Res. 2005; 65:5133-5143.

18. Aderca I, Moser CD, Veerasamy M, Bani-Hani AH, Bonilla-Guerrero R, Ahmed K, Shire A, Cazanave SC, Montoya DP, Mettler TA, Burgart LJ, Nagorney DM, Thibodeau SN, et al. The JNK inhibitor SP600129 enhances apoptosis of HCC cells induced by the tumor suppressor WWOX. J Hepatol. 2008; 49:373-383.

19. Muller PA, Vousden KH. p53 mutations in cancer. Nat Cell Biol. 2013; 15:2-8.

20. Lane DP. Cancer. p53, guardian of the genome. Nature. 1992; 358:15-16.

21. Dotto GP. p21(WAF1/Cip1): more than a break to the cell cycle? Biochim Biophys Acta. 2000; 1471:M43-56.

22. Zhan Q, Chen IT, Antinore MJ, Fornace AJ Jr. Tumor suppressor p53 can participate in transcriptional induction of the GADD45 promoter in the absence of direct DNA binding. Mol Cell Biol. 1998; 18:2768-2778.

23. Miyashita T, Krajewski S, Krajewska M, Wang HG, Lin HK, Liebermann DA, Hoffman B, Reed JC. Tumor suppressor p53 is a regulator of bcl-2 and bax gene expression in vitro and in vivo. Oncogene. 1994; 9:1799-1805.

24. Petak I, Tillman DM, Houghton JA. p53 dependence of Fas induction and acute apoptosis in response to 5-fluorouracil-leucovorin in human colon carcinoma cell lines. Clin Cancer Res. 2000; 6:4432-4441.

25. Bunz F, Hwang PM, Torrance C, Waldman T, Zhang Y, Dillehay L, Williams J, Lengauer C, Kinzler KW, Vogelstein B. Disruption of p53 in human cancer cells alters the responses to therapeutic agents. J Clin Invest. 1999; 104:263-269.

26. Longley DB, Boyer J, Allen WL, Latif T, Ferguson PR, Maxwell PJ, McDermott U, Lynch M, Harkin DP, Johnston PG. The role of thymidylate synthase induction in modulating p53-regulated gene expression in response to 5-fluorouracil and antifolates. Cancer Res. 2002; 62:2644-2649.

27. Zhang L, Yu J, Park BH, Kinzler KW, Vogelstein B. Role of BAX in the apoptotic response to anticancer agents. Science. 2000; 290:989-992.

28. Longley DB, Harkin DP, Johnston PG. 5-fluorouracil: mechanisms of action and clinical strategies. Nat Rev Cancer. 2003; 3:330-338.

29. Li HP, Leu YW, Chang YS. Epigenetic changes in virusassociated human cancers. Cell Res. 2005; 15:262-271.

30. Park IY, Sohn BH, Yu E, Suh DJ, Chung YH, Lee JH, Surzycki SJ, Lee YI. Aberrant epigenetic modifications 
in hepatocarcinogenesis induced by hepatitis $\mathrm{B}$ virus X protein. Gastroenterology. 2007; 132:1476-1494.

31. Lee JO, Kwun HJ, Jung JK, Choi KH, Min DS, Jang KL. Hepatitis B virus X protein represses E-cadherin expression via activation of DNA methyltransferase 1. Oncogene. 2005; 24:6617-6625.

32. Zhong S, Tang MW, Yeo W, Liu C, Lo YM, Johnson PJ. Silencing of GSTP1 gene by CpG island DNA hypermethylation in $\mathrm{HBV}$-associated hepatocellular carcinomas. Clin Cancer Res. 2002; 8:1087-1092.

33. Shim YH, Yoon GS, Choi HJ, Chung YH, Yu E. p16 Hypermethylation in the early stage of hepatitis B virus-associated hepatocarcinogenesis. Cancer Lett. 2003; 190:213-219.

34. Deng YB, Nagae G, Midorikawa Y, Yagi K, Tsutsumi S, Yamamoto S, Hasegawa K, Kokudo N, Aburatani H, Kaneda A. Identification of genes preferentially methylated in hepatitis $\mathrm{C}$ virus-related hepatocellular carcinoma. Cancer Sci. 2010; 101:1501-1510.

35. Rhee I, Bachman KE, Park BH, Jair KW, Yen RW, Schuebel KE, Cui H, Feinberg AP, Lengauer C, Kinzler KW, Baylin SB, Vogelstein B. DNMT1 and DNMT3b cooperate to silence genes in human cancer cells. Nature. 2002; 416:552-556.

36. Guo M, Ren J, House MG, Qi Y, Brock MV, Herman JG. Accumulation of promoter methylation suggests epigenetic progression in squamous cell carcinoma of the esophagus. Clin Cancer Res. 2006; 12:4515-4522.

37. Herman JG, Graff JR, Myohanen S, Nelkin BD, Baylin SB. Methylation-specific, PCR: a novel PCR assay for methylation status of $\mathrm{CpG}$ islands. Proc Natl Acad Sci U S A. 1996; 93:9821-9826.

38. Jia Y, Yang Y, Brock MV, Zhan Q, Herman JG, Guo M. Epigenetic regulation of DACT2, a key component of the Wnt signalling pathway in human lung cancer. J Pathol. 2013; 230:194-204.

39. Yan W, Wu K, Herman JG, Brock MV, Fuks F, Yang L, Zhu H, Li Y, Yang Y, Guo M. Epigenetic regulation of DACH1, a novel Wnt signaling component in colorectal cancer. Epigenetics. 2013; 8:1373-1383.

40. Cregger M, Berger AJ, Rimm DL. Immunohistochemistry and quantitative analysis of protein expression. Arch Pathol Lab Med. 2006; 130:1026-1030.

41. Koo CL, Kok LF, Lee MY, Wu TS, Cheng YW, Hsu JD, Ruan A, Chao KC, Han CP. Scoring mechanisms of p16INK4a immunohistochemistry based on either independent nucleic stain or mixed cytoplasmic with nucleic expression can significantly signal to distinguish between endocervical and endometrial adenocarcinomas in a tissue microarray study. J Transl Med. 2009; 7:25.

42. Pan X, Zhou T, Tai YH, Wang C, Zhao J, Cao Y, Chen Y, Zhang PJ, Yu M, Zhen C, Mu R, Bai ZF, Li HY, et al. Elevated expression of CUEDC2 protein confers endocrine resistance in breast cancer. Nat Med. 2011; 17:708-714.

43. Cao B, Yang Y, Pan Y, Jia Y, Brock MV, Herman JG, Guo M. Epigenetic silencing of CXCL14 induced colorectal cancer migration and invasion. Discov Med. 2013; 16:137-147. 